\title{
TOPOLOGICAL DEGREE APPROACH TO STEADY STATE FLOW
}

\section{Cristina Sburlan}

Department of Mathematics, "Ovidius" University, Bd. Mamaia 124, 8700-Constantza, Romania

C_sburlan@univ-ovidius.ro

\section{Silviu Sburlan}

Department of Mathematics, "Ovidius" University, Bd. Mamaia 124, 8700-Constantza, Romania ssburlan@univ-ovidius.ro

\begin{abstract}
In this paper we study the steady state flow equation, which can be treated as an eigenvalue problem, and we shall apply the results from the topological degree theory and from the bifurcation theory.
\end{abstract}

Keywords: Steady state flow, coincidence degree, bifurcation points

Let $\Omega \subseteq R^{N}\left(\Omega=R^{N}-\mathcal{P}\right), 2 \leq N \leq 3$, be a domain with enough smooth boundary, $Q=\Omega \times(0,+\infty), \Sigma=\partial \Omega \times(0,+\infty)$, with $\mathcal{P}$ a bounded region in $R^{N}$.

We consider the Navier-Stokes system, for the flow of an incompressible fluid:

$$
\begin{gathered}
(\nabla \cdot u)(x, t)=0 \text { (the incompressibility condition }) \\
u_{t}(x, t)+(u \cdot \nabla)(x, t)-\nu \Delta u(x, t)=\nabla p(x, t)+f(x, t),(x, t) \in Q \\
(\text { the Navier }- \text { Stokes flow equation }) \\
u=0 \text { on } \Sigma \text { and } u \rightarrow(1,0,0) \text { for }|x| \rightarrow+\infty .
\end{gathered}
$$

We supose that the body forces are of potential type, i.e.

$$
f(x, t)=\nabla_{x} V(x, t)
$$

The original version of this chapter was revised: The copyright line was incorrect. This has been corrected. The Erratum to this chapter is available at DOI: 10.1007/978-0-387-35690-7_44 
and we note $q:=p+V$, where $p$ is the (unknown) pressure in fluid. Then we can write (2) under the form:

$$
u_{t}(x, t)+(u \cdot \nabla) u(x, t)-\nu \Delta u(x, t)=\nabla q(x, t),
$$

where $\nu$ is the dynamical viscosity $\left(\nu=\frac{1}{R e}\right.$ is the inverse of the Reynolds number). Here, the velocity $u=\left(u_{1}, \ldots, u_{N}\right)$ and the "pressure" $q$ are not known, and they must be determined from the system (1) - (3).

We shall study the case of the steady state flow: $u_{t}=0 \Leftrightarrow u=$ const. The equation (5) becomes:

$$
(u \cdot \nabla) u(x, t)-\nu \Delta u(x, t)=\nabla q(x, t)
$$

Let $X:=\left\{y \in\left(L^{2}(\Omega)\right)^{N} ; \nabla \cdot y=0, y \cdot n=0\right.$ on $\left.\partial \Omega\right\}$ be the Hilbert space of "incompressible fluids" and $E:=\left\{y \in\left(H_{0}^{1}(\Omega)\right)^{N} ; \nabla \cdot y=0\right\}$ be a subspace of $X$, and $P:\left(L^{2}(\Omega)\right)^{N} \rightarrow X$ is Leray projector.

Denote by $A \in L(E, E)$ the Stokes operator:

$$
(A y, w)=\sum_{i=0}^{n} \int_{\Omega} \nabla y_{i} \cdot \nabla w_{i} d x, \forall y, w \in E
$$

and define the nonlinear form:

$$
b(y, z, w):=\sum_{i, j=1}^{N} \int_{\Omega} y_{i} D_{i} z_{j} w_{j} d x
$$

which determines the nonlinear operator $C: E \rightarrow E$ :

$$
C(y, w):=b(y, y, w), \forall y, w \in E .
$$

Then we can reformulate the problem (6) as the problem

$$
\nu A y+C(y)=P(q)
$$

where $A$ is simmetric, i.e. $(A y, w)=(y, A w)$, and strongly monotone, because $(A y, y) \geq\|y\|^{2}$. This is an eigenvalue problem, having $\nu$ as eigenvalue parameter.

Because the embedding $E \hookrightarrow X$ is compact $(2 \leq N \leq 3)$ (from extension of Sobolev theorem for unbounded domains - see [2]), we have that the operator $\widehat{C}: E \subset X \rightarrow X, \widehat{C}=I \circ C$, is compact (the composition of a compact operator with a continuous one). Similary, the operator $\widetilde{A}=I \circ A$ is compact, too.

Using the fact that $\widehat{C}(y, w)$ is. linear in $w$, we can prove that there exists $c>0$ such that:

$$
\|\widehat{C}(y)\| \leq c \cdot\|y\|, \forall y \in E,
$$


where $c$ is the supremum of the "pressure" of fluid.

Denote by $T: X \rightarrow X, T:=P(q)$ and $\widetilde{C}:=\widehat{C}-T$, and by (7), there exists $k>0$ such that:

$$
\|\widetilde{C}(y)\| \leq k \cdot\|y\|, \forall y \in E
$$

The equation (7) can be written:

$$
\nu \widetilde{A} y+\widetilde{C}(y)=0
$$

Now, for two operators $\mathcal{L}: D(\mathcal{L}) \subseteq X \rightarrow X$ and $\mathcal{N}: D(\mathcal{N}) \subseteq X \rightarrow X$, where $\mathcal{L}$ is linear and maximal monotone, and $\mathcal{N}$ is compact, we have that: $\nu \mathcal{L}+\mathcal{N}=I+\nu \mathcal{L}-I+\mathcal{N}=(I+\nu \mathcal{L})\left(I-(I+\nu \mathcal{L})^{-1}(I-\mathcal{N})\right)$.

Because $\mathcal{L}: X \rightarrow X$ is maximal monotone, we have that $I+\nu \mathcal{L}$ is invertible, and the equation $\nu \mathcal{L} y+\mathcal{N}(y)=0$ becomes:

$$
\left(I-(I+\nu \mathcal{L})^{-1}(I-\mathcal{N})\right)(y)=0
$$

Denote by $M(\nu):=(I+\nu \mathcal{L})^{-1}(I-\mathcal{N})$, and remark that this operator is a compact one.

If $D \subset X$ is an open bounded set such that: $\nu \mathcal{L} y+\mathcal{N}(y) \neq 0, \forall y \in \partial D$, then we define the coincidence degree of the pair $(\mathcal{L}, \mathcal{N})$, relatively to $D$ by $d_{\nu}((\mathcal{L}, \mathcal{N}), D):=d_{L S}(I-M(\nu), D, 0)$, where $d_{L S}$ denotes the Leray-Schauder degree. So, this coincidence degree has the properties of the Leray-Schauder degree.

Theorem 1 If $d_{\nu}((\mathcal{L}, \mathcal{N}), D) \neq 0$ then the equation $\nu \mathcal{L} y+\mathcal{N}(y)=0$ has at least one solution in $D$.

Proof. We have that $d_{L S}(I-M(\nu), D, 0) \neq 0$, and from the solution property for the Leray-Schauder degree (see [1]) there exists $y \in D$ such that $(I-M(\nu)) y=0$, and so it results that $\nu \mathcal{L} y+\mathcal{N}(y)=0$.

Theorem 2 Let $\left(\nu_{t}\right)_{t \in[0,1]} \subset(0, \infty)$ be a continuous deformation such that the equation $\nu_{t} \mathcal{L} y+\mathcal{N}(y)=0$ has no solutions $y \in \partial D$, for all $t \in[0,1]$. Then $d_{\nu_{t}}((\mathcal{L}, \mathcal{N}), D)$ is independent of $t \in[0,1]$.

Proof. The conclusion results from the invariance of Leray-Schauder degree to the homotopy (see [1]).

Now, we want to find the bifurcation points of equation (10), i.e. the points $(\nu, 0)$ acumulating nontrivial solutions of $(10)$.

Denote by $\mathcal{C}(\tilde{A})$ the set of all characteristic values of $\widetilde{A}$. Because $\widetilde{A}$ is linear, the algebraic multiplicity of this characteristic values is 1 .

We can state the following result: 
Theorem 3 If $\nu_{0} \in \mathcal{C}(\widetilde{A})$ is such that $\operatorname{dist}\left(\nu_{0}, \mathcal{C}(\widetilde{A}) \backslash\left\{\nu_{0}\right\}\right)>2$, then the equation (10) has at least one bifurcation point in $\left(\nu_{0}-1, \nu_{0}+1\right)$.

Proof. We have that the algebraic multiplicity of $\nu_{0}, m\left(\nu_{0}\right)=1$.

Let $\varepsilon=\operatorname{dist}\left(\nu_{0}, \mathcal{C}(\widetilde{A}) \backslash\left\{\nu_{0}\right\}\right)-2>0, \nu_{1}=\nu_{0}-1-\varepsilon, \nu_{2}=\nu_{0}+1+\varepsilon$.

We have that: $\operatorname{dist}\left(\nu_{1}, \mathcal{C}(\widetilde{A})\right)=\operatorname{dist}\left(\nu_{2}, \mathcal{C}(\widetilde{A})\right)=1+\varepsilon$.

We shall prove that for any $r>0$, the equation (10) has a solution $\left(\nu_{r}, y_{r}\right)$ such that: $\nu_{r} \in\left(\nu_{0}-1, \nu_{0}+1\right)$ and $\left\|y_{r}\right\|=r$.

Let $r>0$ be arbitrary fixed. Assume by contradiction that

$$
\nu \widetilde{A} y+\widetilde{C}(y) \neq 0, \forall\|y\|=r \text { and } \nu \in\left(\nu_{1}, \nu_{2}\right) .
$$

We have that

$$
\begin{aligned}
d_{\nu_{1}}((0, \widetilde{C}), B(0, r)) & =d_{L S}(I-(I-\widetilde{C}), B(0, r), 0)= \\
& =d_{\nu_{2}}((0, \widetilde{C}), B(0, r))
\end{aligned}
$$

Consider the homotopies $H_{t}^{i}=\nu_{i} t\left(\widetilde{A}-\frac{1}{\nu_{i}} I\right)+(1-t) \widetilde{C}, t \in[0,1]$, $i=1,2$. Because $\widetilde{A}: D(\widetilde{A}) \subseteq X \rightarrow X$ is maximal monotone, the operator $\widetilde{A}-\frac{1}{\nu_{i}} I$ is also maximal monotone. We have $\left\|\nu_{i} \widetilde{A} y\right\| \leq\left\|\nu_{i} \widetilde{A} y-y+y\right\| \leq\left\|\nu_{i} \widetilde{A} y-y\right\|+\|y\| \Rightarrow$
$\left\|\nu_{i} \widetilde{A} y-y\right\| \geq\left\|\nu_{i} \widetilde{A} y\right\|-\|y\| \geq \nu_{i}\|y\|-\|y\| \geq \varepsilon \cdot\|y\|>0, \forall y \in B(0, r), i=1,2$.

By the invariance of the coincidence degree to homotopy we have:

$$
d_{\nu_{i}}\left(\left(\widetilde{A}-\frac{1}{\nu_{i}} I, 0\right), B(0, r)\right)=d_{\nu_{i}}((0, \widetilde{C}), B(0, r)), i=1,2 .
$$

We know that $\tilde{A}^{-1}: X \rightarrow X$ is linear, continuous and compact.

From (12) - (13) we obtain

$$
d_{\nu_{1}}\left(\left(\widetilde{A}-\frac{1}{\nu_{1}} I, 0\right), B(0, r)\right)=d_{\nu_{2}}\left(\left(\widetilde{A}-\frac{1}{\nu_{2}} I, 0\right), B(0, r)\right),
$$

i.e.

$$
d_{L S}\left(I-\frac{1}{\nu_{1}} \widetilde{A}^{-1}, B(0, r), 0\right)=d_{L S}\left(I-\frac{1}{\nu_{2}} \widetilde{A}^{-1}, B(0, r), 0\right)
$$

But

$$
d_{L S}\left(I-\frac{1}{\nu_{1}} \widetilde{A}^{-1}, B(0, r), 0\right)=(-1)^{m\left(\nu_{0}\right)} d_{L S}\left(I-\frac{1}{\nu_{2}} \widetilde{A}^{-1}, B(0, r), 0\right)
$$


and $m\left(\nu_{0}\right)=1$, which contradicts (14).

Now, we have that

$$
\nu \widetilde{A}+\widetilde{C}=I+\nu \widetilde{A}-I+\widetilde{C}=(I+\nu \widetilde{A})\left(I-(I+\nu \widetilde{A})^{-1}(I-\widetilde{C})\right),
$$

so, the equation (10) is equivalent to

$$
\begin{gathered}
\left(I-(I+\nu \widetilde{A})^{-1}(I-\widetilde{C})\right)(y)=0 \Leftrightarrow \\
y=(I+\nu \widetilde{A})^{-1}(I-\widetilde{C})(y) \Leftrightarrow \\
y=(I+\nu \widetilde{A})^{-1} y+(I+\nu \widetilde{A})^{-1} \widetilde{C}(y)
\end{gathered}
$$

where the operator $(I+\nu \widetilde{A})^{-1} \widetilde{C}$ is compact, as the composition of a compact operator with a continuos one.

Denoting by $L:=(I+\nu \widetilde{A})^{-1}$ and by $N:=(I+\nu \widetilde{A})^{-1} \widetilde{C}$, the equation (15) becomes

$$
y=L y+N(y),
$$

where $\|N(y)\| \leq c \cdot\|y\|$, with $c>0$.

It is easy to see that $\mu=1$ is a characteristic value for $\widetilde{L}$ and its multiplicity is 1 .

Consider now the eigenvalue problem:

$$
y=\mu \widetilde{L} y+N(y)
$$

Denote by $\mathcal{C}_{0}=\{[\mu, 0] ; \mu \in R\}, \mathcal{S}_{0}$ the set of nontrivial solutions, and $\mathcal{S}:=\overline{\mathcal{S}_{0}}$.

For $\mu=1$, the equation (17) is in fact the equation (16).

Based on the well-known results from the bifurcation theory (see [1]), namely Krasnoselskii and Rabinowitz theorems, we can state the following

Theorem 4 The point $[1,0] \in \mathcal{C}_{0}$ is a bifurcation point for equation (17) .

Proof. Because $\mu=1$ is a characteristic value of $\widetilde{L}$ with odd multiplicity, we can apply Krasnoselskii theorem, and thus $[1,0]$ is a bifurcation point for equation (17).

Theorem 5 Under the above conditions, $\mathcal{S}$ contains a connected component $\mathcal{E}$, passing by $[1,0] \in \mathcal{C}_{0}$, with one of the properties: 
1. $\mathcal{E}$ is unbounded in $R \times X$;

2. $\mathcal{E}$ contains a finite number of points $\left[\mu_{j}, 0\right]$ with $\mu_{j}$ characteristic values of $\widetilde{L}$. Moreover, the number of points with odd multiplicity - including $[1,0]$ - is even.

Proof. Indeed, for $\mu=1$, which has odd multiplicity, the result follows from Rabinowitz theorem.

Comments. Writing Navier-Stokes system as an eigenvalue problem in the case of steady state flow we can deduce not only the existence of the solutions, but also the branche structure of solutions and the bifurcation points. This is perfectly concordant with the physical meaning of steady state flow. Moreover, we see the power of topological methods for solving such problems.

\section{References}

[1] S. Sburlan, Gradul topologic. Lecţii asupra ecuaţiilor neliniare, Ed. Academiei Române, Bucureşti, 1983.

[2] D. Pascali, S. Sburlan, Nonlinear Mappings of Monotone Type, Sijhoff \& Noordhoff Int. Publ., 1978.

[3] C. Sburlan, S. Sburlan, Abstract Fourier Method for Stokes Equation, Bull. PAMM-2039, 2002 (to appear).

[4] S. Sburlan, C. Mortici, A Coincidence Degree for Bifurcation Problems, BAM1784/2001 XCIV, 2000.

[5] S. Sburlan, C. Mortici, Topogical Methods for Semilinear Problems with Maximal Monotone Nonlinearity, Conference at 26th Congress of American Romanian Academy of Arts and Sciences, July 25-29, Montreal, 2001.

[6] S. Sburlan, L. Barbu and C. Mortici, Ecuaţii Diferenţiale, Integrale şi Sisteme Dinamice, Ed. Ex Ponto, Constanţa, 1999.

[7] S. Sburlan, G. Morosanu, Monotonicity Methods for Partial Differential Equations, MB-11/PAMM, Budapest, 1999.

[8] R. Temam, Navier-Stokes Equations, North-Holland Publishing Company, 1977. 